\title{
Label-free, single molecule detection of cytokines using optical microcavities
}

\author{
Andrea M. Armani ${ }^{1}$, Scott E. Fraser ${ }^{1,2}$ and Kerry J. Vahala ${ }^{1}$ \\ Department of Applied Physics, California Institute of Technology, 1200 E California Blvd, Pasadena, CA 91125 \\ Department of Biology, California Institute of Technology, 1200 E California Blvd, Pasadena, CA 91125 \\ armani@caltech.edu, sefraser@caltech.edu,vahala@caltech.edu
}

Interleukin-2 (IL2) is a cytokine that regulates T-cell growth and is used in cancer therapies. By sensitizing a microcavity sensor surface with anti-IL2 and monitoring the resonant frequency, single molecules of IL2 can be detected.

(C) 2007 Optical Society of America

OCIS codes: 230.5750 Resonators, 230.0040 Detectors

Ultra-high-Q micro-resonators have recently demonstrated very sensitive and specific chemical and biological detection. $[1,2]$ The extreme sensitivity relies upon a thermo-optic effect that uses resonant enhancement of the optical probe wave [1]. In particular, the binding of molecules to the surface of the resonant cavity creates a local "hot spot" that induces a refractive index change, and, in turn, increases the resonant wavelength of the microcavity. Beyond sensitivity, detection specificity is achieved through surface functionalization or attaching antibodies to the surface of the resonant cavity.

In the present work, planar arrays of ultra-high-Q microtoroid resonators $[3,4]$ were used to perform label-free, single molecule detection of Interleukin-2 (IL-2), a cytokine released in response to immune system activation by extrinsic and intrinsic stimuli (Fig. 1a). The surface of the microtoroids was sensitized for detection using a Protein G protocol: 1) immerse toroids in water, 2) inject $0.1 \mu \mathrm{M}$ Protein $\mathrm{G}$ to form a monolayer, 3) inject $0.1 \mu \mathrm{M} \mathrm{IL-2}$ antibody and 4) inject IL-2 antigen. The Protein G binds to the Fc region of the antibody, thereby orienting the antibodies on the surface of the resonant cavity. Additional solutions in serum were also made to verify the sensor's application in a more realistic environment. Several IL-2 concentrations were used, ranging from 100aM to 900aM. The resonant wavelength was monitored in real-time on an oscilloscope and downloaded directly to a computer. The data acquisition rate, solution injection rate and IL-2 concentration were optimized to allow single molecule binding events to be resolved. Tapered optical fiber waveguides were used to couple $680 \mathrm{~nm}$ light into and out of the microtoroid sensor (Fig. 1a, inset).

During the initial series of label-free detection experiments, the pure IL-2 solutions were injected into the volume surrounding the microtoroid.[1] The solutions were injected continuously into the bath surrounding the microtoroid using a syringe pump. The resonant wavelength was automatically determined every 0.2 seconds and recorded. As seen in Figure 1b, the microtoroid was able to detect individual IL-2 molecules at these concentration levels, as indicated by the staircase steps seen. The variability in the step size seen is a result of molecules binding throughout the whispering gallery mode.

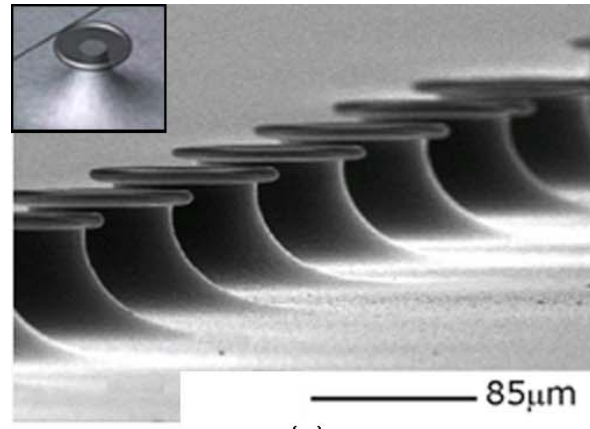

(a)

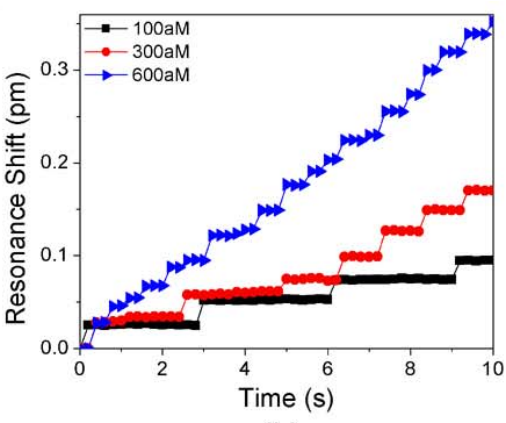

(b)

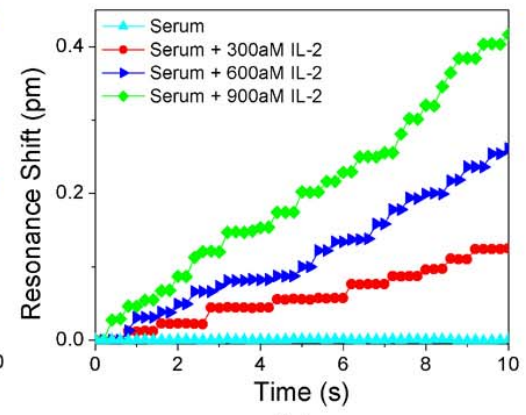

(c)

Fig. 1: Toroidal microcavity sensor. a) A scanning electron micrograph (SEM) of an array of microtoroid resonant sensors. Inset: Artistic rendering of the microtoroid coupled to a tapered optical fiber waveguide. b) Detection of 100aM of IL2 (black squares), 300aM of Il-2 (red circles), 600aM IL-2 (blue triangles) as resonant wavelength shift versus time. The individual binding events are seen in this graph as steps. c) Detection performed in serum containing 300aM of Il-2 (red circles), 600aM IL-2 (blue triangles), and 900aM IL-2 (green diamonds). An additional detection experiment was performed in pure serum (cyan triangle) with a null result. The individual binding events are seen in this graph as steps. 
A complimentary experiment was performed to investigate the robustness of the sensor to contaminants in the volume around the sensor. A series of IL-2 concentrations in serum were prepared at [300,600, 900]aM. As can be seen in Figure 1c, individual binding events can still be resolved; therefore, the serum did not have a significant impact on the detection.

Other experiments which were performed and which will be presented involved alternative surface sensitization techniques and detection in the presence of Tryptophan. The theoretical basis for detection, the label-free, single molecule IL-2 experiments in buffer and in serum, and the surface functionalization techniques will be presented.

[1] A. M. Armani, R. P. Kulkarni, S. E. Fraser, R. C. Flagan, and K. J. Vahala, "Label-Free, Single-Molecule Detection with Optical Microcavities," Science, 2007.

[2] A. M. Armani and K. J. Vahala, "Heavy water detection using ultra-high-Q microcavities," Optics Letters, vol. 31, pp. 1896-1898, 2006.

[3] A. M. Armani, D. K. Armani, B. Min, K. J. Vahala, and S. M. Spillane, "Ultra-high-Q microcavity operation in $\mathrm{H}_{2} \mathrm{O}$ and $\mathrm{D}_{2} \mathrm{O}$," Applied Physics Letters, vol. 87, pp. 151118, 2005.

[4] D. K. Armani, T. J. Kippenberg, S. M. Spillane, and K. J. Vahala, "Ultra-high-Q toroid microcavity on a chip," Nature, vol. 421, pp. 925-928, 2003. 